PROCEEDINGS OF THE

AMERICAN MATHEMATICAL SOCIETY

Volume 130, Number 8 , Pages 2451-2459

S 0002-9939(02)06446-8

Article electronically published on February 4, 2002

\title{
ON THE CELLULAR DECOMPOSITION OF THE EXCEPTIONAL LIE GROUP $G_{2}$
}

\author{
MAMORU MIMURA AND TETSU NISHIMOTO \\ (Communicated by Paul Goerss)
}

\begin{abstract}
The present note is to give a cellular decomposition of the compact connected exceptional Lie group $G_{2}$.
\end{abstract}

\section{INTRODUCTION}

Let us denote by $G_{2}$ the compact connected exceptional Lie group of rank 2 . By definition $G_{2}$ is the automorphism group $\operatorname{Aut}(\mathfrak{C})$, where $\mathfrak{C}$ is the Cayley algebra. It has been long known that $G_{2}$ has the homotopy type of

$$
S^{3} \cup e^{5} \cup e^{6} \cup e^{8} \cup e^{9} \cup e^{11} \cup e^{14} .
$$

The purpose of the present note is to give a cellular decomposition of $G_{2}$ :

$$
G_{2}=S^{3} \cup e^{5} \cup e^{6} \cup e^{8} \cup e^{9} \cup e^{11} \cup e^{14} .
$$

\section{PRELIMINARY}

Let us recall known results on $G_{2}$ which will be needed later.

The Cayley algebra $\mathfrak{C}$ is isomorphic to $\mathbb{R}^{8}$ as an $\mathbb{R}$-module and we denote its basis by $\left\{e_{0}, e_{1}, e_{2}, e_{3}, e_{4}, e_{5}, e_{6}, e_{7}\right\}$. Notice that the multiplication is not associative. The element $e_{0}$ is the unit of the algebra which we denote by 1 . The multiplication of the remaining basis is given in the following diagram:

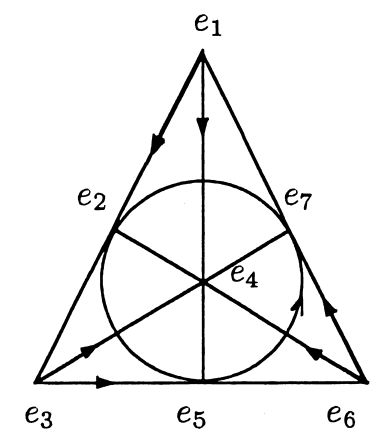

Received by the editors March 1, 2001.

2000 Mathematics Subject Classification. Primary 57N60.

Key words and phrases. Exceptional Lie groups, cellular decomposition.

This work was partially supported by the Grant-in-Aid for Scientific Research \#12640025 from the Japan Society of Promotion of Science. 
Then the exceptional Lie group $G_{2}$ is defined to be

$$
G_{2}=\{g \in S O(8) \mid g(x) g(y)=g(x y), x, y \in \mathfrak{C}\}=\operatorname{Aut}(\mathfrak{C})
$$

Since $g(1)=1$ for any $g \in G_{2}$, we may regard $G_{2}$ as a subgroup of $S O(7)$. So from now on, we express an element of $G_{2}$ as that of $S O(7)$. The subgroup of elements in $G_{2}$ fixing $e_{1}$ is known to be isomorphic to $S U(3)$. The subgroup of elements in $G_{2}$ fixing $e_{1}$ and $e_{2}$ is known to be isomorphic to $S U(2)$. Thus we regard $S U(3)$ and $S U(2)$ as subgroups of $G_{2}$. Let $S^{6}$ be the unit sphere of $\mathbb{R}^{7}$ whose basis is $\left\{e_{i} \mid 1 \leq i \leq 7\right\}$ and $S^{5}$ be the unit sphere of $\mathbb{R}^{6}$ whose basis is $\left\{e_{i} \mid 2 \leq i \leq 7\right\}$. Then there are two principal fibre bundles over them:

$$
\begin{aligned}
& S U(3) \longrightarrow G_{2} \stackrel{p_{1}}{\longrightarrow} S^{6}, \\
& S U(2) \longrightarrow S U(3) \stackrel{p_{2}}{\longrightarrow} S^{5},
\end{aligned}
$$

where $p_{i}(g)=g\left(e_{i}\right)$ for $i=1,2$. Let $H$ be the subgroup of $G_{2}$ defined by

$$
H=G_{2} \cap(S O(3) \oplus S O(4)) .
$$

Lemma 2.1. $h g h^{-1} \in S U(2)$ for any $h \in H$ and $g \in S U(2)$.

Proof. It is obvious, since $S U(2)=G_{2} \cap(\{1\} \oplus S O(4))$, where $\{1\}$ denotes the subgroup of $S O(3)$ consisting of the identity element.

In the remainder of the section, we will construct cells of $G_{2}$. Let $D^{i}(1 \leq i \leq 3)$ be the $i$-dimensional discs defined respectively by

$$
\begin{aligned}
& D^{3}=\left\{\left(x_{1}, x_{2}, x_{3}\right) \in \mathbb{R}^{3} \mid x_{1}^{2}+x_{2}^{2}+x_{3}^{2} \leq 1\right\}, \\
& D^{2}=\left\{\left(y_{1}, y_{2}\right) \in \mathbb{R}^{2} \mid y_{1}^{2}+y_{2}^{2} \leq 1\right\}, \\
& D^{1}=\left\{z_{1} \in \mathbb{R} \mid z_{1}^{2} \leq 1\right\} .
\end{aligned}
$$

We define $V^{3}, V^{5}$ and $V^{6}$ as follows:

$$
V^{3}=D^{3}, \quad V^{5}=D^{3} \times D^{2}, \quad V^{6}=D^{3} \times D^{2} \times D^{1},
$$

and put $X, Y$ and $Z$ as

$$
X=\sqrt{1-x_{1}^{2}-x_{2}^{2}-x_{3}^{2}}, \quad Y=\sqrt{1-y_{1}^{2}-y_{2}^{2}}, \quad Z=\sqrt{1-z_{1}^{2}} .
$$


We define three maps $A, B, C$ respectively of $D^{i}$ to $G_{2}$ for $1 \leq i \leq 3$ as follows:

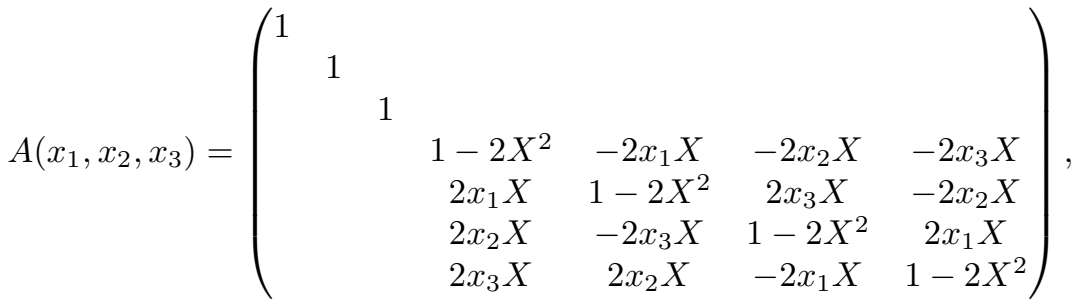

$$
\begin{aligned}
& B\left(y_{1}, y_{2}\right)=\left(\begin{array}{ccccccc}
1 & & & & & \\
& y_{1} & -y_{2} & -Y & 0 & & \\
& y_{2} & y_{1} & 0 & -Y & & \\
Y & 0 & y_{1} & y_{2} & & \\
0 & Y & -y_{2} & y_{1} & & \\
& & & & & 1 & \\
& & & & & & 1
\end{array}\right) \\
& C\left(z_{1}\right)=\left(\begin{array}{ccccccc}
z_{1} & 0 & -Z & & & & \\
0 & 1 & 0 & & & & \\
Z & 0 & z_{1} & & & & \\
& & & 1 & & & \\
& & & & z_{1} & 0 & -Z \\
& & & & 0 & 1 & 0 \\
& & & & Z & 0 & z_{1}
\end{array}\right)
\end{aligned}
$$

where blanks consist of the zero element. After having prepared these definitions, we will construct some cells of $G_{2}$. Let $\varphi_{i}$ be a map of $V^{i}$ to $G_{2}$ for $i=3,5,6$ defined respectively by

$$
\begin{aligned}
& \varphi_{3}\left(x_{1}, x_{2}, x_{3}\right)=A\left(x_{1}, x_{2}, x_{3}\right), \\
& \varphi_{5}\left(x_{1}, x_{2}, x_{3}, y_{1}, y_{2}\right)=B\left(y_{1}, y_{2}\right) A\left(x_{1}, x_{2}, x_{3}\right) B\left(y_{1}, y_{2}\right)^{-1}, \\
& \varphi_{6}\left(x_{1}, x_{2}, x_{3}, y_{1}, y_{2}, z_{1}\right)=C\left(z_{1}\right) B\left(y_{1}, y_{2}\right) A\left(x_{1}, x_{2}, x_{3}\right) B\left(y_{1}, y_{2}\right)^{-1} C\left(z_{1}\right)^{-1} .
\end{aligned}
$$

We define eight cells $e^{j}$ for $j=0,3,5,6,8,9,11,14$ as follows:

$$
\begin{aligned}
& e^{0}=\{1\}, \quad e^{3}=\operatorname{Im} \varphi_{3}, \quad e^{5}=\operatorname{Im} \varphi_{5}, \quad e^{6}=\operatorname{Im} \varphi_{6}, \\
& e^{8}=e^{5} e^{3}, \quad e^{9}=e^{6} e^{3}, \quad e^{11}=e^{6} e^{5}, \quad e^{14}=e^{6} e^{5} e^{3} .
\end{aligned}
$$

We denote the boundary and the interior of a cell $e^{i}$ simply by $\dot{e}^{i}$ and by $e^{i}$ respectively.

\section{A cellular decomposition of $S U(3)$}

Yokota [Y1], Y3] constructed a cellular decomposition of $S U(n)$. In this section, we reconstruct a cellular decomposition of $S U(3)$ for our purpose, which is essentially the same as Yokota's decomposition.

As is known, $S U(2)$ is homeomorphic to $S^{3}$, and hence $e^{0} \cup e^{3}$ is a cellular decomposition of $S U(2)$.

Lemma 3.1. The composite map $p_{2} \varphi_{5}:\left(V^{5}, \partial V^{5}\right) \rightarrow\left(S^{5},\left\{e_{2}\right\}\right)$ is a relative homeomorphism. 
Proof. We express the map $\left.\left(p_{2} \varphi_{5}\right)\right|_{V^{5} \backslash \partial V^{5}}$ as follows:

$$
\left(\begin{array}{c}
0 \\
a_{2} \\
a_{3} \\
a_{4} \\
a_{5} \\
a_{6} \\
a_{7}
\end{array}\right)=p_{2} \varphi_{5}\left(x_{1}, x_{2}, x_{3}, y_{1}, y_{2}\right)=\left(\begin{array}{c}
0 \\
1-2 X^{2} Y^{2} \\
2 x_{1} X Y^{2} \\
2 Y\left(y_{1} X^{2}-x_{1} y_{2} X\right) \\
-2 Y\left(x_{1} y_{1} X+y_{2} X^{2}\right) \\
-2 x_{2} X Y \\
-2 x_{3} X Y
\end{array}\right)
$$

and hence

$$
\left(\begin{array}{c}
0 \\
1-a_{2} \\
a_{3} \\
a_{4} \\
a_{5} \\
a_{6} \\
a_{7}
\end{array}\right)=2 X Y\left(\begin{array}{c}
0 \\
X Y \\
x_{1} Y \\
y_{1} X-x_{1} y_{2} \\
-x_{1} y_{1}-y_{2} X \\
-x_{2} \\
-x_{3}
\end{array}\right)
$$

Since $X>0, Y>0$ and $1-a_{2}>0$, an easy calculation from the second component in the above equation gives the following equation:

$$
X Y=\frac{\sqrt{1-a_{2}}}{\sqrt{2}},
$$

from which we easily obtain

$$
\begin{aligned}
& x_{2}=\frac{-a_{6}}{\sqrt{2\left(1-a_{2}\right)}}, \\
& x_{3}=\frac{-a_{7}}{\sqrt{2\left(1-a_{2}\right)}} .
\end{aligned}
$$

Further we obtain two more equalities from the above equation:

$$
\begin{aligned}
& \left(1-a_{2}\right)^{2}+a_{3}^{2}=4 X^{2} Y^{4}\left(x_{1}^{2}+X^{2}\right), \\
& a_{4}^{2}+a_{5}^{2}=4 X^{2} Y^{2}\left(y_{1}^{2}+y_{2}^{2}\right)\left(x_{1}^{2}+X^{2}\right)=4 X^{2} Y^{2}\left(1-Y^{2}\right)\left(x_{1}^{2}+X^{2}\right) .
\end{aligned}
$$

Using these two equalities, we obtain

$$
Y^{2}=\frac{\left(1-a_{2}\right)^{2}+a_{3}^{2}}{\left(1-a_{2}\right)^{2}+a_{3}^{2}+a_{4}^{2}+a_{5}^{2}} .
$$

It follows from (3.1) and (3.4) that

$$
X^{2}=\frac{\left(1-a_{2}\right)\left(\left(1-a_{2}\right)^{2}+a_{3}^{2}+a_{4}^{2}+a_{5}^{2}\right)}{2\left(\left(1-a_{2}\right)^{2}+a_{3}^{2}\right)} .
$$

It follows from (3.2), (3.3) and (3.5) that

$$
x_{1}^{2}=\frac{a_{3}^{2}\left(\left(1-a_{2}\right)^{2}+a_{3}^{2}+a_{4}^{2}+a_{5}^{2}\right)}{2\left(1-a_{2}\right)\left(\left(1-a_{2}\right)^{2}+a_{3}^{2}\right)} .
$$

Since $Y>0$, (3.4) implies that

$$
Y=\frac{\sqrt{\left(1-a_{2}\right)^{2}+a_{3}^{2}}}{\sqrt{\left(1-a_{2}\right)^{2}+a_{3}^{2}+a_{4}^{2}+a_{5}^{2}}} .
$$


Since $X>0$, (3.5) implies that

$$
X=\frac{\sqrt{\left(1-a_{2}\right)\left(\left(1-a_{2}\right)^{2}+a_{3}^{2}+a_{4}^{2}+a_{5}^{2}\right)}}{\sqrt{2\left(\left(1-a_{2}\right)^{2}+a_{3}^{2}\right)}} .
$$

Since the signs of $x_{1}$ and $a_{3}$ are the same, (3.6) implies that

$$
x_{1}=\frac{a_{3} \sqrt{\left(1-a_{2}\right)^{2}+a_{3}^{2}+a_{4}^{2}+a_{5}^{2}}}{\sqrt{2\left(1-a_{2}\right)\left(\left(1-a_{2}\right)^{2}+a_{3}^{2}\right)}} .
$$

Now we determine $y_{2}$; we have

$$
a_{4} x_{1}+a_{5} X=-2 X Y\left(x_{1}^{2}+X^{2}\right) y_{2} .
$$

Substituting the equations (3.1), (3.8) and (3.9) in the above equation, we obtain

$$
y_{2}=\frac{-a_{3} a_{4}-\left(1-a_{2}\right) a_{5}}{\sqrt{\left(\left(1-a_{2}\right)^{2}+a_{3}^{2}\right)\left(\left(1-a_{2}\right)^{2}+a_{3}^{2}+a_{4}^{2}+a_{5}^{2}\right)}} \text {. }
$$

Finally we determine $y_{1}$; we have

$$
a_{4} X-a_{5} x_{1}=2 X Y\left(x_{1}^{2}+X^{2}\right) y_{1} .
$$

Substituting the equations (3.1), (3.8) and (3.9) in the above equation, we obtain

$$
y_{1}=\frac{\left(1-a_{2}\right) a_{4}-a_{3} a_{5}}{\sqrt{\left(\left(1-a_{2}\right)^{2}+a_{3}^{2}\right)\left(\left(1-a_{2}\right)^{2}+a_{3}^{2}+a_{4}^{2}+a_{5}^{2}\right)}} .
$$

Thus we have expressed $x_{1}, x_{2}, x_{3}, y_{1}, y_{2}$ in terms of $a_{2}, \cdots, a_{7}$, that is, the inverse map has been constructed, which completes the proof.

Proposition 3.2. $e^{0} \cup e^{3} \cup e^{5} \cup e^{8}$ is a cellular decomposition of $S U(3)$.

Proof. First we will show that $e^{i} \cap e^{j}=\emptyset$ if $i \neq j$. We consider the following three cases:

(1) For the case where $i=0$ and $j=3$, it is obvious that $e^{0} \cap e^{3}=\emptyset$ since $e^{0} \cup e^{3}$ is a cellular decomposition of $S U(2)$.

(2) For the case where $i \in\{0,3\}$ and $j \in\{5,8\}$, we have $p_{2}\left(e^{i}\right)=e_{2}$ and $p_{2}\left(e^{j}\right)=$ $S^{5} \backslash\left\{e_{2}\right\}$. Then we have $e^{i} \cap \dot{e}^{j}=\emptyset$.

(3) For the case where $i=5$ and $j=8$, suppose that $A \in e^{5} \cap e^{8}$. Since $e^{8}=e^{5} \dot{e}^{3}$, we can put $A=A_{1} A_{2}$ where $A_{1} \in e^{5}$ and $A_{2} \in e^{3}$. We have $A=A_{1}$ since $p_{2}(A)=p_{2}\left(A_{1} A_{2}\right)=p_{2}\left(A_{1}\right)$ and $\left.p_{2}\right|_{e^{5}}$ is monic. Then we have $A_{2}=1 \in \dot{e}^{3}$, which is a contradiction. Thus $e^{5} \cap e^{8}=\emptyset$.

Next, we will check that the boundaries of the cells are included in the lower dimensional cells. It is obvious that the boundary $\dot{e}^{3}$ is included in $e^{0}$. Observe that the boundary $\dot{e}^{5}$ is a union of the following two sets:

$$
\begin{aligned}
& \left\{B A B^{-1} \mid A \in A\left(\dot{D}^{3}\right), B \in B\left(D^{2}\right)\right\}, \\
& \left\{B A B^{-1} \mid A \in A\left(D^{3}\right), B \in B\left(\dot{D}^{2}\right)\right\} .
\end{aligned}
$$

The first set contains only the identity element since $A$ is the identity element. Lemma 2.1 implies that the second set is contained in $S U(2)$ since $B$ is contained in $H$. Thus we have $\dot{e}^{5} \subset e^{3}$. Further we have $\dot{e}^{8}=\dot{e}^{5} e^{3} \cup e^{5} \dot{e}^{3} \subset e^{3} e^{3} \cup e^{5} e^{0}=e^{3} \cup e^{5}$.

Finally, we will show that the inclusion map $e^{0} \cup e^{3} \cup e^{5} \cup e^{8} \rightarrow S U(3)$ is epic. Let $g \in S U(3)$. If $p_{2}(g)=e_{2}$, then $g$ is contained in $S U(2)=e^{0} \cup e^{3}$. Suppose that $p_{2}(g) \neq e_{2}$. There is an element $h \in e^{5}$ such that $p_{2}(h)=p_{2}(g)$. Then we have 
$h^{-1} g \in S U(2)=e^{0} \cup e^{3}$, since $p_{2}\left(h^{-1} g\right)=e_{2}$. Therefore we have $g \in h\left(e^{0} \cup e^{3}\right) \subset$ $e^{0} \cup e^{3} \cup e^{5} \cup e^{8}$.

\section{A Cellular Decomposition of $G_{2}$}

First we need to show

Lemma 4.1. The composite $\operatorname{map} p_{1} \varphi_{6}:\left(V^{6}, \partial V^{6}\right) \rightarrow\left(S^{6},\left\{e_{1}\right\}\right)$ is a relative homeomorphism.

Proof. We express the map $\left.\left(p_{1} \varphi_{6}\right)\right|_{V^{6} \backslash \partial V^{6}}$ as follows:

$$
\left(\begin{array}{l}
a_{1} \\
a_{2} \\
a_{3} \\
a_{4} \\
a_{5} \\
a_{6} \\
a_{7}
\end{array}\right)=p_{1} \varphi_{6}\left(x_{1}, x_{2}, x_{3}, y_{1}, y_{2}, z_{1}\right)=\left(\begin{array}{c}
1-2 X^{2} Y^{2} Z^{2} \\
2 x_{1} X Y^{2} Z \\
2 z_{1} X^{2} Y^{2} Z \\
-2 X Y Z\left(x_{1} y_{1}+y_{2} X\right) \\
-2 X Y Z\left(y_{1} z_{1} X-x_{1} y_{2} z_{1}+x_{2} Z\right) \\
-2 X Y Z x_{3} \\
-2 X Y Z\left(y_{1} X Z-x_{1} y_{2} Z-x_{2} z_{1}\right)
\end{array}\right)
$$

and hence

$$
\left(\begin{array}{c}
1-a_{1} \\
a_{2} \\
a_{3} \\
a_{4} \\
a_{5} \\
a_{6} \\
a_{7}
\end{array}\right)=2 X Y Z\left(\begin{array}{c}
X Y Z \\
x_{1} Y \\
z_{1} X Y \\
-x_{1} y_{1}-y_{2} X \\
-y_{1} z_{1} X+x_{1} y_{2} z_{1}-x_{2} Z \\
-x_{3} \\
-y_{1} X Z+x_{1} y_{2} Z+x_{2} z_{1}
\end{array}\right)
$$

We set for simplicity

$$
\begin{gathered}
\alpha_{1}=\left(1-a_{1}\right)^{2}+a_{3}^{2}, \quad \alpha_{2}=\left(1-a_{1}\right)^{2}+a_{2}^{2}+a_{3}^{2}, \quad \alpha_{3}=\left(1-a_{1}\right)^{2}+a_{2}^{2}+a_{3}^{2}+a_{4}^{2}, \\
\beta_{1}=a_{3} a_{5}+\left(1-a_{1}\right) a_{7}, \quad \beta_{2}=a_{2} a_{4}+a_{3} a_{5}+\left(1-a_{1}\right) a_{7} .
\end{gathered}
$$

Since $1-a_{1}>0$, we have $\alpha_{i}>0$ for $i=1,2,3$. By an easy calculation one can obtain the following three equations:

$$
\begin{aligned}
& Z^{2}=\frac{\left(1-a_{1}\right)^{2}}{\alpha_{1}}, \\
& X^{2} Y^{2}=\frac{1-a_{1}}{2 Z^{2}}=\frac{\alpha_{1}}{2\left(1-a_{1}\right)}, \\
& z_{1}^{2}=1-Z^{2}=\frac{a_{3}^{2}}{\alpha_{1}} .
\end{aligned}
$$

Since $Z \geq 0$ and $1-a_{1} \geq 0$, 4.1) implies that

$$
Z=\frac{1-a_{1}}{\sqrt{\alpha_{1}}} .
$$

Since the signs of $z_{1}$ and $a_{3}$ are the same, (4.3) implies that

$$
z_{1}=\frac{a_{3}}{\sqrt{\alpha_{1}}}
$$


We easily have

$$
x_{3}=\frac{-a_{6}}{2 X Y Z}=\frac{-a_{6}}{\sqrt{2\left(1-a_{1}\right)}} .
$$

Next we determine $X$ and $Y$; we have

$$
\begin{gathered}
\left(a_{4}^{2}+\left(a_{5} z_{1}+a_{7} Z\right)^{2}\right) Y^{2}=4 X^{2} Y^{4} Z^{2}\left(y_{1}^{2}+y_{2}^{2}\right)\left(x_{1}^{2}+X^{2}\right) \\
=4 X^{2} Y^{4} Z^{2}\left(1-Y^{2}\right)\left(x_{1}^{2}+X^{2}\right), \\
a_{2}^{2}\left(1-Y^{2}\right)=4 X^{2} Y^{4} Z^{2}\left(1-Y^{2}\right) x_{1}^{2} .
\end{gathered}
$$

It follows from these two equalities that

$$
\left(a_{4}^{2}+\left(a_{5} z_{1}+a_{7} Z\right)^{2}\right) Y^{2}-a_{2}^{2}\left(1-Y^{2}\right)=4 X^{4} Y^{4} Z^{2}\left(1-Y^{2}\right) .
$$

Substituting the equations (4.2), (4.4) and (4.5) in the above equation, we obtain

$$
a_{4}^{2} Y^{2}+\frac{\left(a_{3} a_{5}+\left(1-a_{1}\right) a_{7}\right)^{2}}{\alpha_{1}} Y^{2}+a_{2}^{2} Y^{2}-a_{2}^{2}=\alpha_{1}\left(1-Y^{2}\right),
$$

whence we have

$$
Y^{2}=\frac{\alpha_{1} \alpha_{2}}{\alpha_{1} \alpha_{3}+\beta_{1}^{2}}
$$

Since $Y \geq 0$, we have

$$
Y=\frac{\sqrt{\alpha_{1} \alpha_{2}}}{\sqrt{\alpha_{1} \alpha_{3}+\beta_{1}^{2}}}
$$

Since $X \geq 0$, (4.2) and (4.7) imply that

$$
X=\frac{\sqrt{\alpha_{1} \alpha_{3}+\beta_{1}^{2}}}{\sqrt{2\left(1-a_{1}\right) \alpha_{2}}} .
$$

We easily have

$$
x_{1}=\frac{a_{2}}{2 X Y^{2} Z}=\frac{a_{2} \sqrt{\alpha_{1} \alpha_{3}+\beta_{1}^{2}}}{\sqrt{2\left(1-a_{1}\right) \alpha_{1} \alpha_{2}}} .
$$

Now we determine $y_{1}$ and $y_{2}$; we have

$$
a_{4} x_{1}+a_{5} z_{1} X+a_{7} X Z=-2 X Y Z\left(x_{1}^{2}+X^{2}\right) y_{1},
$$

into which substituting (4.4), (4.5), (4.7), (4.8) and (4.9) we obtain

$$
y_{1}=\frac{-\beta_{2} \sqrt{\alpha_{1}}}{\sqrt{\alpha_{2}\left(\alpha_{1} \alpha_{3}+\beta_{1}^{2}\right)}} .
$$

Quite similarly the equation

$$
a_{4} X-a_{5} x_{1} z_{1}-a_{7} x_{1} Z=-2 X Y Z\left(x_{1}^{2}+X^{2}\right) y_{2}
$$

gives rise to

$$
y_{2}=\frac{a_{2} \beta_{1}-a_{4} \alpha_{1}}{\sqrt{\alpha_{2}\left(\alpha_{1} \alpha_{3}+\beta_{1}^{2}\right)}} .
$$

Finally we determine $x_{2}$; we have

$$
a_{5} Z-a_{7} z_{1}=-2 X Y Z x_{2},
$$


which gives

$$
x_{2}=\frac{a_{3} a_{7}-\left(1-a_{1}\right) a_{5}}{\sqrt{2\left(1-a_{1}\right) \alpha_{1}}} .
$$

Thus we have expressed $x_{1}, x_{2}, x_{3}, y_{1}, y_{2}, z_{1}$ in terms of $a_{1}, \cdots, a_{7}$, that is, the inverse map has been constructed and this completes the proof.

The following is our main result.

Theorem 4.2. The cell complex $e^{0} \cup e^{3} \cup e^{5} \cup e^{6} \cup e^{8} \cup e^{9} \cup e^{11} \cup e^{14}$ thus constructed gives a cellular decomposition of $G_{2}$.

Proof. First we show that $e^{i} \cap e^{j}=\emptyset$ if $i \neq j$. We consider the following three cases:

(1) For the case where $i, j \in\{0,3,5,8\}$, both cells $e^{i}$ and $e^{j}$ are in $S U(3)$ and $e^{0} \cup e^{3} \cup e^{5} \cup e^{8}$ is a cellular decomposition of $S U(3)$. Then we have $e^{i} \cap e^{j}=\emptyset$ if $i \neq j$.

(2) For the case where $i \in\{0,3,5,8\}$ and $j \in\{6,9,11,14\}$, we have $p_{1}\left(e^{i}\right)=\left\{e_{1}\right\}$ and $p_{1}\left(e^{j}\right)=S^{6} \backslash\left\{e_{1}\right\}$. Then we have $e^{i} \cap e^{j}=\emptyset$.

(3) For the case where $i, j \in\{6,9,11,14\}$, suppose that $A \in \dot{e}^{i} \cap \dot{e}^{j}$. Since $e^{i}=$ $\dot{e}^{\circ} e^{i-6}$ and $e^{j}=e^{\circ} e^{j-6}$, we can put $A=A_{1} A_{2}=A_{1}^{\prime} A_{2}^{\prime}$ where $A_{1}, A_{1}^{\prime} \in e^{6}$, $A_{2} \in \mathrm{e}^{i-6}$ and $A_{2}^{\prime} \in \mathrm{e}^{j-6}$. We have $A_{1}=A_{1}^{\prime}$, since $p_{1}\left(A_{1}\right)=p_{1}\left(A_{1} A_{2}\right)=$ $p_{1}\left(A_{1}^{\prime} A_{2}^{\prime}\right)=p_{1}\left(A_{1}^{\prime}\right)$ and $\left.p_{1}\right|_{e^{\circ}}$ is monic. Then we have $A_{2}=A_{2}^{\prime}$ and the first case shows that $i-6=j-6$, that is, $i=j$. Thus $\dot{e}^{i} \cap \dot{e}^{j}=\emptyset$ if $i \neq j$.

Next, we will check that the boundaries of the cells are included in the lower dimensional cells. In the proof of Proposition 3.2 it is proved that the boundaries $\dot{e}^{3}, \dot{e}^{5}$ and $\dot{e}^{8}$ are included in the lower dimensional cells. Observe that the boundary $\dot{e}^{6}$ is a union of the following three sets:

$$
\begin{aligned}
& \left\{C B A B^{-1} C^{-1} \mid A \in A\left(\dot{D}^{3}\right), B \in B\left(D^{2}\right), C \in C\left(D^{1}\right)\right\}, \\
& \left\{C B A B^{-1} C^{-1} \mid A \in A\left(D^{3}\right), B \in B\left(\dot{D}^{2}\right), C \in C\left(D^{1}\right)\right\}, \\
& \left\{C B A B^{-1} C^{-1} \mid A \in A\left(D^{3}\right), B \in B\left(D^{2}\right), C \in C\left(\dot{D}^{1}\right)\right\} .
\end{aligned}
$$

The first set contains only the identity element, since $A$ is the identity element. Lemma 2.1 implies that the second set is contained in $S U(2)$, since $B$ and $C$ are contained in the subgroup $H$. We consider the third set. If $C=C(1)=1$, it is obvious that $C B A B^{-1} C^{-1}=B A B^{-1} \in e^{5}$. Suppose that $C=C(-1)$. It is easy to check that

$$
\begin{aligned}
& C B\left(y_{1}, y_{2}\right) C^{-1}=B\left(y_{1},-y_{2}\right), \\
& C A\left(x_{1}, x_{2}, x_{3}\right) C^{-1}=A\left(-x_{1}, x_{2},-x_{3}\right) .
\end{aligned}
$$

Thus the third set is contained in $e^{5}$, since we have

$$
\begin{aligned}
& C B\left(y_{1}, y_{2}\right) A\left(x_{1}, x_{2}, x_{3}\right) B\left(y_{1}, y_{2}\right)^{-1} C^{-1} \\
= & \left(C B\left(y_{1}, y_{2}\right) C^{-1}\right)\left(C A\left(x_{1}, x_{2}, x_{3}\right) C^{-1}\right)\left(C B\left(y_{1}, y_{2}\right)^{-1} C^{-1}\right) \\
= & B\left(y_{1},-y_{2}\right) A\left(-x_{1}, x_{2},-x_{3}\right) B\left(y_{1},-y_{2}\right)^{-1} .
\end{aligned}
$$

We have $\dot{e}^{9}=e^{6} \dot{e}^{3} \cup \dot{e}^{6} e^{3} \subset e^{6} e^{0} \cup e^{5} e^{3}=e^{6} \cup e^{8}$. We also have $\dot{e}^{11}=\dot{e}^{6} e^{5} \cup e^{6} \dot{e}^{5} \subset$ $e^{5} e^{5} \cup e^{6} e^{3}=e^{5} \cup e^{9}$, and $\dot{e}^{14}=\dot{e}^{6} e^{5} e^{3} \cup e^{6} \dot{e}^{5} e^{3} \cup e^{6} e^{5} \dot{e}^{3} \subset e^{5} e^{5} e^{3} \cup e^{6} e^{3} e^{3} \cup e^{6} e^{5}=$ $e^{8} \cup e^{9} \cup e^{11}$. 
Finally, we will show that the inclusion map $e^{0} \cup e^{3} \cup e^{5} \cup e^{6} \cup e^{8} \cup e^{9} \cup e^{11} \cup e^{14} \rightarrow G_{2}$ is epic. Let $g \in G_{2}$. If $p_{1}(g)=e_{1}$, then $g$ is contained in $S U(3)=e^{0} \cup e^{3} \cup e^{5} \cup e^{8}$. Suppose that $p_{1}(g) \neq e_{1}$. There is an element $h \in e^{6}$ such that $p_{1}(h)=p_{1}(g)$. Thus we have $h^{-1} g \in S U(3)=e^{0} \cup e^{3} \cup e^{5} \cup e^{8}$ since $p_{1}\left(h^{-1} g\right)=e_{1}$. Therefore we have $g \in h\left(e^{0} \cup e^{3} \cup e^{5} \cup e^{8}\right) \subset e^{0} \cup e^{3} \cup e^{5} \cup e^{6} \cup e^{8} \cup e^{9} \cup e^{11} \cup e^{14}$.

\section{REFERENCES}

[A] J. F. Adams, Lectures on exceptional Lie groups, (edited by Zafer Mahmud and Mamoru Mimura) Chicago Lectures in Mathematics. University of Chicago Press, Chicago, IL, 1996. MR 98b:22001

[Y1] I. Yokota, On the cell structures of $\mathrm{SU}(n)$ and $\mathrm{Sp}(n)$, Proc. Japan Acad., 31 (1955), 673677. MR 17:774a

[Y2] I. Yokota, On the cellular decompositions of unitary groups, J. Inst. Polytech. Osaka City Univ. Ser. A., 7 (1956), 39-49. MR 18:918e

[Y3] I. Yokota, Groups and Topology, Shokabo, (1971) (in Japanese).

Department of Mathematics, Okayama University, Okayama, Japan 700-8530

E-mail address: mimura@math.okayama-u.ac.jp

Kinki Welfare University, Fukusaki-Cho Hyogo, Japan 679-2217

E-mail address: nishimoto@kinwu.ac.jp 